\title{
Esofagitis eosinofílica: reporte de un caso
}

\section{A case report of eosinophilic esophagitis}

\author{
Pedro Rosales Torres, ${ }^{1}$ Rafael Pila Pérez, ${ }^{2}$ Rafael Pila Peláez, ${ }^{3}$ Pedro León Acosta, ${ }^{4}$ Yudenia Toledo Cabarcos.${ }^{5}$
}

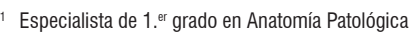
e Instructor, Hospital Provincial Docente Manuel
Ascunce Domenech, Camagüey, Cuba. e Instructor, Hospital Provincial Docente Manuel
Ascunce Domenech, Camagüey, Cuba.

2 Especialista de 2. do grado en Medicina Interna y Profesor Titular, Hospital Provincial Docente Manuel Ascunce Domenech, Camagüey, Cuba.

3 Especialista de 2. do grado en Medicina Interna y Profesor Auxiliar, Hospital Provincial Docente Manue Ascunce Domenech, Camagüey, Cuba.

4 Especialista de 1. ${ }^{\text {er }}$ grado en Medicina Interna Instructor, Hospital Provincial Docente Manuel Ascunce Domenech, Camagüey, Cuba.

5 Especialista de 1. er grado en Anatomía Patológica e Instructora, Hospital Provincial Docente Manuel Ascunce Domenech, Camagüey, Cuba.
}

*Correspondencia: leon@mad.cmw.sld.cu

\section{Resumen}

Objetivo: presentar un caso infrecuente de un paciente con esofagitis eosinofilica, el cual constituye el primer caso reportado en nuestro país en 53 años. Caso clínico: paciente de 28 años, género femenino, de raza blanca, con antecedentes personales de atopia, rinitis alérgica, dermatitis, diarreas ocasionales y asma, por la cual ha estado ingresada en varias ocasiones; además presenta antecedentes familiares de asma bronquial. Refiere que estas alteraciones comenzaron a los 17 años, y desde alli fue asistida por varios especialistas. Hace 10 meses presentó disfagia, la cual fue en aumento, dolor torácico y abdominal, pirosis y pérdida de peso. En el examen físico presentó sibilancias diseminadas en ambos campos pulmonares. El estudio analítico completo y los exámenes imagenológicos resultaron normales. La endoscopia y la biopsia esofágica mostraron los elementos compatibles con esofagitis eosinofilica. El tratamiento se realizó con esteroides orales, monteleukast e inhibidores de la bomba de protones, que mostraron resultados excelentes. Conclusiones: la esofagitis eosinofilica es una enfermedad de la que todavía existe un gran desconocimiento. Es más común en niños, hombres jóvenes de raza blanca, y la cual, por lo regular, tiene un componente atópico marcado. En la adultez se manifiesta por disfagia e impactación de los alimentos. Para el diagnóstico de una esofagitis eosinofilica tiene que haber síntomas de disfunción esofágica, más de 15 eosinófilos por campo, falta de respuesta a los inhibidores de la bomba de protones y exclusión del reflujo gastroesofágico. Dependiendo de cada caso, el paciente debe ser tratado de forma multidisciplinaria por gastroenterólogos, alergólogos, inmunólogos y nutriólogos.

\section{Palabras clave \\ Esofagitis eosinofilica.}

\section{Abstract}

Objective: We present the first case of eosinophilic esophagitis (EE) reported in our country in fifty-three years. Clinical Case: The patient was 28-year-old white woman with a personal history of atopy, allergic rhinitis, dermatitis and occasional diarrhea and asthma. She had relatives with bronchial asthma and had been admitted to hospitals several times previously. According to the patient, these alterations began at age 17 , and she had seen several specialists since that time. Ten months prior to this admission, increasingly severe dysphagia accompanied by chest and abdominal pain, heartburn and weight loss began. Upon physical examination, wheezing was evident in both lung fields. The complete analytical study and imaging tests were all normal. Endoscopy and esophageal biopsy showed elements compatible with eosinophilic esophagitis. Excellent results were obtained from treatment with oral steroids, montelukast and proton pump inhibitors. Conclusions: $\mathrm{EE}$ is still poorly understood. It is more common in children and young white men, and there is usually a marked atopic component. In adulthood it is manifested by dysphagia and impaction of food. To diagnose $\mathrm{EE}$ there must be symptoms of esophageal dysfunction, more than 15 eosinophils per field, lack of response to proton pump inhibitors and exclusion of gastroesophageal reflux. Depending on the case, patients may require multidisciplinary treatment by gastroenterologists, allergists, immunologists and nutritionists. 


\section{INTRODUCCIÓN}

La esofagitis eosinofílica es resultado de la inflamación del esófago por eosinófilos, un tipo de leucocito que participa en la inmunidad frente a ciertas infecciones (especialmente por parásitos), y en muchas enfermedades alérgicas como el asma bronquial (1). Aunque es una enfermedad poco habitual, la frecuencia de la esofagitis eosinofílica está aumentando en muchos países desarrollados; las regiones no están claras, pero se conoce que todos los tipos de alergias son ahora más frecuentes que en las generaciones pasadas (2), además por el estudio de otras patologías que presentan infiltración eosinofílica, como la esofagitis infecciosa, la enfermedad de Crohn, el síndrome hipereosinofílico, las reacciones adversas a medicamentos y la enfermedad del tejido conectivo (1). Se han utilizado diferentes términos para referirse a esta entidad, pero el término más aceptado es el de esofagitis eosinofílica (3).El primer caso reportado en la literatura corresponde a Dobbins y colaboradores en 1977, quienes señalaron el caso de un adulto con antecedentes de atopia que presentaba espasmo esofágico.

La esofagitis eosinofílica es una enfermedad de impacto mundial, de la cualse han descrito casos en todos los continentes menos en África (5). Se estima que la prevalencia actual varía entre el 0,4-1 \% en pacientes adultos y entre el 0,04-0,09 \% en niños, con una incidencia anual de 1:100 000 en adultos y 1:10 000 en población pediátrica; los adultos afectados son hombres jóvenes entre 20-50 años, con una edad promedio de 38 años, y una proporción de 3:1 respecto a las mujeres. Los síntomas reportados son similares entre ambos sexos (5). El objetivo de este trabajo es presentar el caso de una paciente con esofagitis eosinofílica, el cual es el primero en nuestro país en 53 años.

\section{CASO CLÍNICO}

Paciente femenina de 28 años, blanca, maestra, no fumadora, con antecedentes familiares de alergia y asma. Refiere que, desde la adolescencia, a los 17 años, comienza a presentar atopias, rinitis alérgica, dermatitis atópicas recurrentes, diarreas ocasionales y asma bronquial, por lo que ingresó en varias ocasiones. Ha sido estudiada por los servicios de neumología, inmunología y alergia, donde se le practicó una prueba por punción cutánea o prick test $(\mathrm{PT})$, la cual mostró sensibilidad para las lacticinas, pan, frijoles, arroz y tomate; al practicar estudio de inmunoglobulina $\mathrm{E}$ alérgeno-especifica ( $\mathrm{IgE}$ ), esta resultó elevada para estos alimentos, por lo que los alergólogos la trataron con múltiples antihistamínicos y una dieta, lo cual le brindó una mejoría durante un buen tiempo. A los 10 meses comienza con disfagia que ha ido en aumento, pirosis, dolor torácico y abdominal epigástrico, por lo que acude a consulta y se ingresa para su estudio.

\section{Examen físico}

La paciente está decaída, pero no un estado general deficiente. Se encuentra hidratada, su piel y sus mucosas están normocoloreadas, y sus faneras no muestran alteraciones. No presenta adenopatías periféricas, ni edemas. No tiene lesiones en la piel. En la auscultación pulmonar se encontró que su frecuencia respiratoria es de 16 respiraciones por minuto (rpm). Presenta murmullo vesicular disminuido con sibilancias diseminadas en ambos campos pulmonares. En la auscultación cardíaca se encontró que la paciente presenta latidos cardíacos rítmicos y golpeados, sin soplos. Su presión arterial es de $110 / 70 \mathrm{~mm} \mathrm{Hg}$.

$\mathrm{Al}$ tacto, el abdomen está blando, depresible, no presenta dolor, y no hay signos de visceromegalia. El resto del examen físico, incluyendo el ginecológico, no presenta alteraciones. El fondo de ojo es normal.

\section{Pruebas}

Todos los estudios bioquímicos y hematológicos se encontraron dentro de límites normales. Por su parte, la radiografía de tórax, el electrocardiograma (ECG) y la radiografía de esófago, estómago y duodeno resultaron normales. El ultrasonido abdominal, ginecológico y la tomografía axial computarizada (TAC) no mostraron alteraciones.

En la esofagogastroduodenoscopia se muestran estenosis focales y presencia de pápulas puntiformes superficiales con evidente inflamación, anillos circunferenciales (esófago felino) con exudados puntiformes abundantes. El estómago y el duodeno se encuentran normales.

El estudio histológico muestra una inflamación con numerosos eosinófilos intraepiteliales con extensa granulación (más numerosos en la mitad superior del epitelio) y la formación de microabcesos en la superficie (Figuras 1-4). El estudio histológico de estómago y duodeno resulta normal.

Se comienza un tratamiento con dieta blanda en forma de puré, caldos, sopas, y se eliminan todos los alimentos que desencadenaban las crisis. Se añade omeprazol, monteleukast y prednisona durante ocho semanas, los cuales, tras su administración, brindan una respuesta favorable. Posteriormente se realiza una nueva esofagogastroduodenoscopia que resulta normal.

\section{DISCUSIÓN}

En condiciones normales, el esófago es un órgano carente de eosinófilos, por lo que la presencia de estos es conside- 


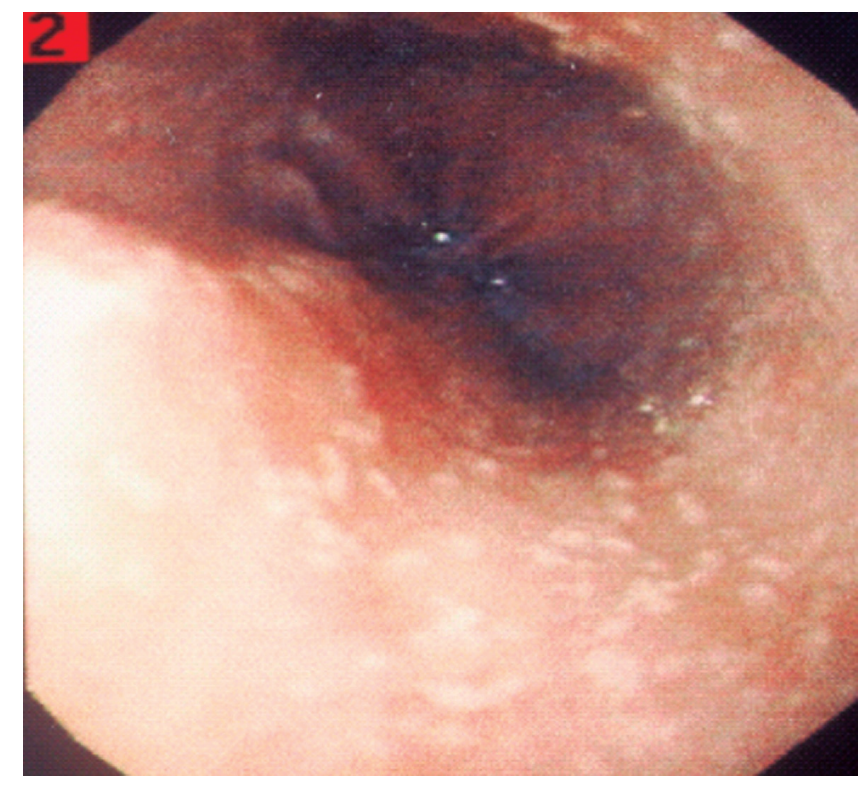

Figura 1. Esofagogastroduodenoscopia que muestra las típicas pápulas puntiformes blanquecinas.

rada como patológica. Hasta hace unos años, la presencia de eosinófilos en la mucosa esofágica era considerada un marcador de reflujo (1). En los últimos años se han descrito un gran número de enfermos con síntomas de disfunción esofágica y gran infiltración eosinofílica en el esófago, quienes no responden a medidas antirreflujo y presentan un $\mathrm{pH}$ esofágico normal, lo cual corresponde a una esofagitis eosinofílica (3). Noel y colaboradores, en un estudio de 103 pacientes (entre 0-19 años) con esofagitis eosinofílica, reportaron un predominio del género masculino (71\%) con antecedentes de atopia con la mitad de ellos y antecedentes familiares de atopia en un $70 \%$; nuestro caso tenía antecedentes personales y familiares de atopia, la cual se presenta en el $60 \%$ y en el $20-40 \%$ de los casos, respectivamente (6). Los factores descritos en la fisiopatología de la esofagitis eosinofílica son la atopia, en la cual se señalan algunos alimentos y aeroalergenos; la eotaxina-3, que implica un componente genético; las interleucinas, como mediadoras de la inflamación; y el reflujo gastroesofágico, como un componente en discusión (3). Se ha señalado que la presencia de los eosinófilos en el esófago es patológica y con tendencia a la cronicidad; sin embargo, lo que se ignora en la actualidad es la causa de la infiltración eosinofílica del esófago (1). Las manifestaciones son muy variadas entre cada paciente y según sus edades; algunos presentan síntomas de manera constante y en otros aparecen de modo intermitente o de forma estacional (2). La disfagia y la impactación alimentaria son más frecuentes en adultos,

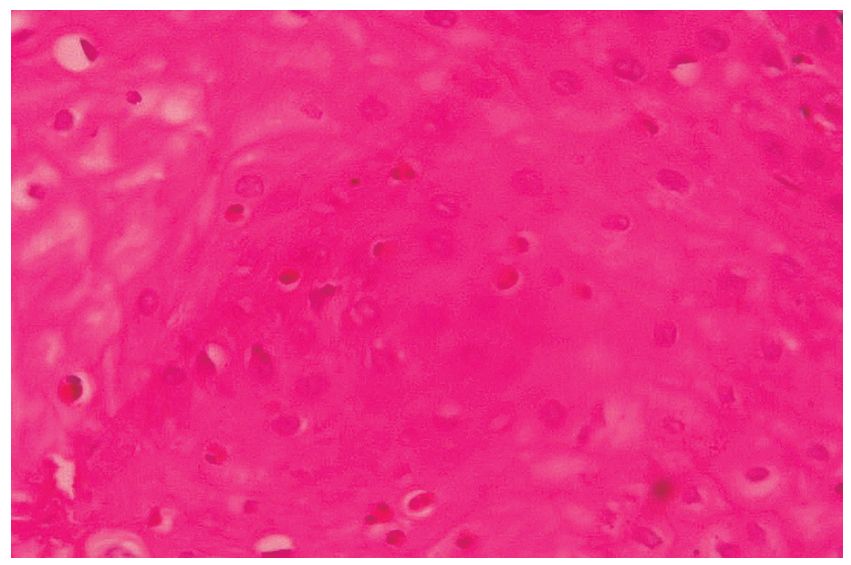

Figura 2. Microfotografía que muestra el epitelio mucoso esofágico con presencia de eosinófilos de distribución difusa. H/E 20 x.

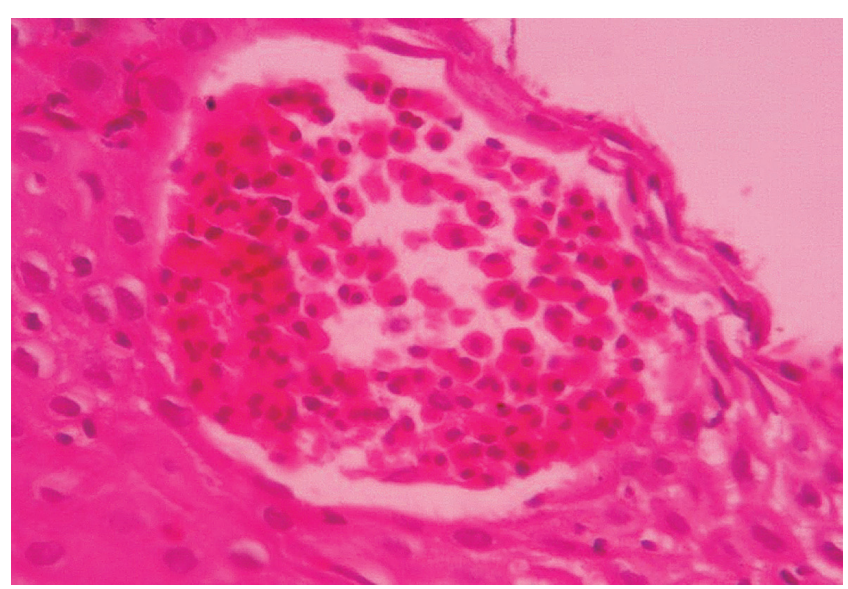

Figura 3. Campo histológico correspondiente a pápulas puntiformes blanquecinas. Obsérvese la formación de un absceso eosinofílico en el tercio superior del epitelio. H/E $40 \mathrm{x}$.

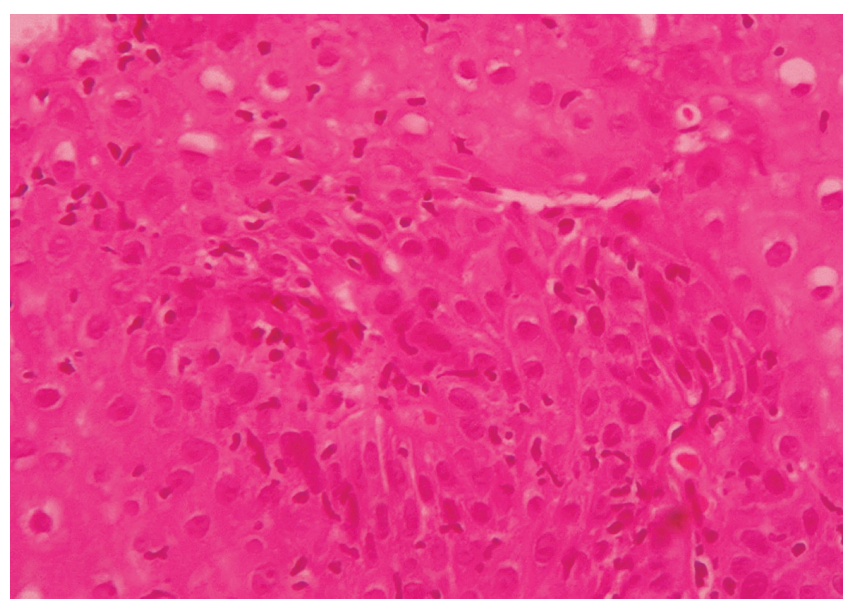

Figura 4. Histología en la que la distribución de las células eosinofílicas es más profunda e irregular hacia el tercio medio e inferior del esófago. $\mathrm{H} / \mathrm{E} 40 \mathrm{x}$. 
seguido de síntomas de reflujo gastroesofágico, dolor torácico y abdominal (1-3). En un estudio realizado por Desai y colaboradores se encontró que la esofagitis eosinofílica era responsable del $50 \%$ de las impactaciones alimentarias esofágicas en consulta ambulatoria (7). Se señala un retraso importante en el diagnóstico de la enfermedad en la población adulta, con un promedio de 4,5 años desde el inicio de los síntomas hasta el momento del diagnóstico (1); en este caso fue superior. En la mayor parte de los enfermos (50-80\%), tanto adultos como niños, se presenta alguna condición atópica: asma, sinusitis, lesiones dérmicas, alergia alimentaria o eccema, los cuales se observan con los cambios de estación $(1,3,5)$, tal como se presentó en esta paciente, teniendo en cuenta los antecedentes genéticos de la misma. Entre las complicaciones se señalan las alteraciones estructurales esofágicas, el síndrome de Boerhaave y las deficiencias nutricionales (8).

El diagnóstico se confirma con endoscopia y toma de biopsia, en las que se observan más de 15 eosinófilos por campo $(1,2)$, de manera similar a como se constató en esta paciente. Los hallazgos endoscópicos más comunes son: surcos esofágicos, anillos esofágicos, granulaciones blanquecinas y estenosis esofágicas; las placas blanquecinas están asociadas con la presencia de microabscesos eosinofílicos y las áreas de gran densidad de infiltrado de eosinófilos (2). El $30 \%$ de las endoscopias pueden parecer normales, por lo que se debe tener cuidado durante las mismas debido a la fragilidad de la mucosa. Se recomienda intentar tomar cinco biopsias y realizar una biopsia de estómago y duodeno con el objetivo de descartar otras entidades (2), así como se realizó en esta paciente.

El tratamiento es controversial, por lo que no se ha definido cuál es la terapéutica óptima, así como tampoco las metas esperadas $(1,5,7)$. Liacouras y colaboradores observaron en 381 pacientes que la asociación entre esteroides y la dieta mejoraba significativamente los síntomas y hallazgos histológicos, e iguales resultados mostraron con esta terapéutica (9). Para Teltelbaum y colaboradores (10) y Konikoff y colaboradores (11), los esteroides pueden utilizarse sistémica o tópicamente durante 4-6 semanas; por nuestra parte, los utilizamos por vía oral durante 8 semanas, y mantuvimos una pauta descendente durante 6 meses.

La dieta consiste en eliminar todos los alimentos sólidos por fórmulas nutricionales adecuadas, y evitar los alimentos identificados como alérgenos (1-3, 6-10). Debido a la relación de la esofagitis eosinofílica con el reflujo gastroesofágico se ha planteado la utilización de inhibidores de la bomba de protones como tratamiento primario o coadyuvante, los cuales se utilizaron en este caso. La recomendación actual es que los inhibidores de la bomba de protones no deben ser considerados como la terapia primaria (3). Los leucotrienos son sustancias quimioatrayentes de los eosinófilos, y se postularon como otra opción terapéutica, ya que con ellos se ha encontrado una mejoría de los síntomas, pero no de la histología, por lo que estos medicamentos no son recomendados en la esofagitis eosinofílica (12).. En la actualidad se han planteado terapias que actuán contra sustancias específicas identificadas dentro de la cascada inflamatoria, como el caso del mepolizumab, un anticuerpo monoclonal humanizado contra la interleucina 5 (IL-5) (13). Nosotros empleamos los esteroides junto a un inhibidor de la bomba de protones y un antagonista de leucotrienos, con los cuales obtuvimos resultados favorables.

\section{CONCLUSIONES}

La esofagitis eosinofílica es una entidad que consiste en una inflamación del esófago que se caracteriza por la presencia de un número elevado de eosinófilos en los estudios histológicos. Es un trastorno de origen alérgico y su frecuencia está aumentando en la población, debido a que se tiene un mejor conocimiento al respecto. La fisiopatología no es conocida con certeza y no existe, por tanto, un tratamiento definitivo. El diagnóstico se confirma con la endoscopia y con la toma de cinco biopsias, incluyendo una de estómago y duodeno. El diagnóstico diferencial debe realizarse con enfermedades más frecuentes que la esofagitis eosinofílica, y que tienen infiltración eosinofílica del esófago.

\section{Conflicto de intereses}

Los autores señalan que este trabajo no ha sido financiado o manipulado de cualquier otra forma que pudiera llevar un conflicto de intereses.

\section{REFERENCIAS}

1. Furuta GT, Liacouras CA, Collins MH, Gupta SK, Justinich C, Putnam PE, et al. Eosinophilic esophagitis in children and adults: a systematic review and consensus recommendations for diagnosis and treatment. Gastroenterology. 2007 Oct;133(4):1342-63. doi: 10.1053/j.gastro.2007.08.017.

2. González G, Torres J, Molina R, Harris P. Esofagitis eosinofílica en niños: características clínicas y endoscópicas. RevMed Chile 2009;137:666-671. doi: 10.4067/S003498872009000500010.

3. Beltrán C, García R, Espino A, Silva C. Esofagitis eosinofilica: una enfermedad emergente. Rev Otorrinolaringol Cir Cabeza y cuello. 2009;69:287-298. doi: 10.4067/S071848162009000300013.

4. Dobbins JW, Sheahan DG, Behar J. Eosinophilic gastroenteritis with esophageal involvement. Gastroenterology. 1977 Jun;72(6):1312-6.

5. Buckmeier BK, Rothenberg ME, Collins NH. The incidence and prevalence of eosinophilicesophagitis. J 
AllergyclinInmunol 2008; 121(2 Suppl 1):S71. doi: 10.1016/j.jaci.2007.12.281.

6. Noel RJ, Putnam PE, Rothenberg ME. Eosinophilic esophagitis. N Engl J Med. 2004 Aug 26;351(9):940-1. doi: 10.1056/NEJM200408263510924.

7. Desai TK, Stecevic V, Chang CH, Goldstein NS, Badizadegan K, Furuta GT. Association of eosinophilic inflammation with esophageal food impaction in adults. Gastrointest Endosc. 2005 Jun;61(7):795-801. doi: 10.1016/s00165107(05)00313-5.

8. Gupte AR, Draganov PV. Eosinophilic esophagitis. World J Gastroenterol. 2009 Jan 7;15(1):17-24. doi: 10.3748/ wjg.15.17.

9. Liacouras CA, Wenner WJ, Brown K, Ruchelli E. Primary eosinophilic esophagitis in children: successful treatment with oral corticosteroids. J Pediatr Gastroenterol Nutr. 1998 Apr;26(4):380-5. doi: 10.1097/00005176-19980400000004.

10. Teitelbaum JE, Fox VL, Twarog FJ, Nurko S, Antonioli D, Gleich G, et al. Eosinophilic esophagitis in children: immu- nopathological analysis and response to fluticasone propionate. Gastroenterology. 2002 May;122(5):1216-25. doi: 10.1053/gast.2002.32998.

11. Konikoff MR, Noel RJ, Blanchard C, Kirby C, Jameson $\mathrm{SC}$, Buckmeier BK, et al. A randomized, double-blind, placebo-controlled trial of fluticasone propionate for pediatric eosinophilic esophagitis. Gastroenterology. 2006 Nov;131(5):1381-91. doi: 10.1053/j.gastro.2006.08.033.

12. Ferré-Ybarz L, Nevot Falcó S, Plaza-Martín AM. Eosinophilic oesophagitis: clinical manifestations and treatment options. The role of the allergologist. Allergol Immunopathol (Madr). 2008 Nov-Dec;36(6):358-65. doi: 10.1016/S0301-0546(08)75869-5.

13. Stein ML, Collins MH, Villanueva JM, Kushner JP, Putnam PE, Buckmeier BK, Filipovich AH, Assa'ad AH, Rothenberg ME. Anti-IL-5 (mepolizumab) therapy for eosinophilic esophagitis. J Allergy Clin Immunol. 2006 Dec;118(6):1312-9. doi: 10.1016/j.jaci.2006.09.007. 